\title{
Forced Convection Heat Transfer from a Heated Cylinder in an Axial Background Flow in a Porous Medium: Three Exactly Solvable Cases
}

\author{
Eugen Magyari
}

Received: 9 July 2012 / Accepted: 15 November 2012 / Published online: 30 November 2012

C Springer Science+Business Media Dordrecht 2012

\begin{abstract}
Assuming a background flow of velocity $U=U(x)$ in the axial direction $x$ of a circular cylinder with surface temperature distribution $T_{w}=T_{w}(x)$ in a saturated porous medium, for the temperature boundary layer occurring on the cylinder three exactly solvable cases are identified. The functions $\left\{U(x), T_{w}(x)\right\}$ associated with these cases are given explicitly, and the corresponding exact solutions are expressed in terms of the modified Bessel function $K_{0}(z)$, the incomplete Gamma function $\Gamma(a, z)$ and the confluent hypergeometric function $U(a, b, z)$, respectively. The correlation between the Nusselt number and the Péclet number as well as the curvature effects on the heat transfer are discussed in all these cases in detail. Some "universal" features of the exponential surface temperature distribution are also pointed out.
\end{abstract}

Keywords Porous media · Forced convection · Exact solution · Nusselt number · Péclet number $\cdot$ Curvature parameter

\section{Introduction}

The majority of the published papers dealing with convective heat transfer in porous media are concerned with the external natural convection case. The second place in this ranking can be assigned to the mixed convection studies. Surprisingly, the forced convection case which is the very basic form of the convection heat transfer, in spite of its numerous industrial and geothermic applications, has received rather little attention. The above ranking holds in external flow in general, but also in the case of convection from circular cylinders revisited in the present paper.

The pioneering work on the free convection heat transfer from a heated vertical cylinder (embedded in a saturated Darcy porous medium) has been published nearly four decades ago by Minkowycz and Cheng (1976). In this paper, it has been shown that in the boundary layer

E. Magyari $(\bowtie)$

Departement Physik, Theoretische Physik, Universität Basel, Klingelbergstr. 82, 4056 Basel, Switzerland e-mail: magyari@bluewin.ch 
approximation, an exact similarity reduction of the problem becomes possible only in the linear case $\lambda=1$ of the considered surface temperature distributions of the power-law form $T_{w}=T_{\infty}+A x^{\lambda}$ ( $x$ denotes the axial coordinate). In other cases, approximate local and nonlocal similarity solutions were reported. Twenty years later, the problem of Minkowycz and Cheng (1976) has been revisited by Bassom and Rees (1996), who have presented asymptotic analyses and numerical solutions of the problem by the Keller-box method.

The related mixed convection problem has mainly been examined in the case of an isothermal cylinder aligned parallel to a uniform background flow. The existence domain and the features of the corresponding boundary layer solutions both in the aiding and opposing flow regimes has been investigated by Merkin and Pop (1987). The non-similarity solutions of this problem have been studied by Hooper et al. (1994), while by Pop and $\mathrm{Na}$ (1998) the case of variable surface heat flux has been considered. More recently, a special exact solution for the mixed convection on the vertical cylinder has been reported by Magyari et al. (2005), assuming that both the velocity of the background flow and the surface temperature distribution of the cylinder are linear functions of the axial coordinate $x$. Further references to the above addressed topics can be found in the comprehensive work of Nield and Bejan (2006).

A scanning of the pertinent literature led us to the surprising result that the forced convection heat transfer from a heated cylindrical body aligned parallel to a background flow in a saturated porous medium has been addressed until now in a single research work only. It is the paper of Vasantha et al. (1987) in which the case of an isothermal cylinder in a uniform axial stream has been considered. In this case, similarly to the corresponding free and mixed convection problems, an exact similarity reduction of the governing equations is not possible. Accordingly, no exact analytical solution can exist in this case. Thus, we arrived to the question to find out whether other types of background velocity and surface temperature distributions $\left\{U(x), T_{w}(x)\right\}$ could exist, so that the problem of the longitudinal forced convection on the heated cylinder admits exact analytical solutions at all. The answer on this question is that there exist exactly three types of such distributions $\left\{U(x), T_{w}(x)\right\}$ which are specified by Eqs. (5)-(7) below. The goal of the present paper is to write down the corresponding exact solutions explicitly, and to discuss the pertinent heat transfer characteristics in detail. This will be accomplished in the subsequent sections of the paper.

\section{Basic Equations and Problem Formulation}

Consider an impermeable semi-infinite circular cylinder of radius $r_{0}$ and surface temperature distribution $T_{w}=T_{w}(x)$ embedded in a Darcy porous medium. The cylinder is aligned with its axis parallel to a background flow of velocity $U=U(x)$ and uniform temperature $T_{\infty}$ which was already set up in the medium. The geometrical set-up, the coordinate system, the boundary conditions, and the temperature boundary layer are shown schematically in Fig. 1.

The equations governing this forced convection boundary layer flow are [see e.g., Vasantha et al. 1987 with $U_{\infty}$ replaced by $\left.U=U(x)\right]$

$$
\begin{gathered}
\frac{\partial}{\partial x}(r u)+\frac{\partial}{\partial r}(r v)=0 \\
u=U(x) \\
u \frac{\partial T}{\partial x}+v \frac{\partial T}{\partial r}=\frac{\alpha_{m}}{r} \frac{\partial}{\partial r}\left(r \frac{\partial T}{\partial r}\right)
\end{gathered}
$$

with the boundary conditions

$$
v=0, T=T_{w}(x) \text { on } r=r_{0} \text {, and } T \rightarrow T_{\infty} \text { as } r \rightarrow \infty \text {. }
$$




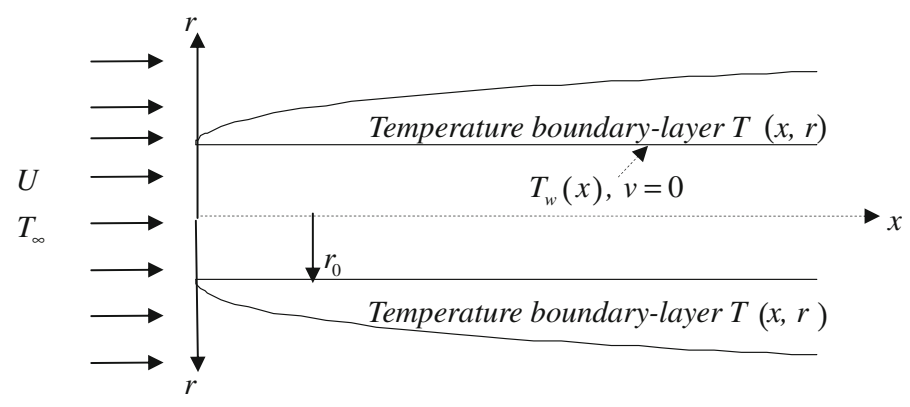

Fig. 1 Sketch of the coordinate system, boundary conditions, and flow domain in the porous medium

Here $x \geq 0$ and $r \geq r_{0}$ are the axial and radial coordinates, respectively, $u$ and $v$ are the velocity components in the $x$ and $r$ directions, $T=T(x, r)$ stands for the temperature field, and $\alpha_{m}$ denotes the equivalent thermal diffusivity of the saturated porous medium.

The goal of the present paper is to find such stream velocity and surface temperature distributions $\left\{U(x), T_{w}(x)\right\}$ for which the boundary value problem (1)-(4) admits exact solutions, and to give these solutions in terms of known higher transcendental functions which allow in turn for a comprehensive discussion of the heat transfer characteristics of the flow. Three such distributions $\left\{U(x), T_{w}(x)\right\}$ will be identified, namely

$$
\begin{aligned}
& U(x)=U_{0}, \quad T_{w}(x)=T_{\infty}+T_{0} \exp \left(a \frac{x}{r_{0}}\right), \quad a>0 \\
& U(x)=U_{0} \frac{x}{r_{0}}, \quad T_{w}(x)=T_{\infty}+T_{0} \\
& U(x)=U_{0} \frac{x}{r_{0}}, \quad T_{w}(x)=T_{\infty}+T_{0} \frac{x}{r_{0}} .
\end{aligned}
$$

Here $U_{0}$ is the uniform velocity of the background flow in case (i) and its local velocity at the axial distance $x=r_{0}$ from the leading edge in cases (ii) and (iii). $T_{0}$ is the excess temperature of the surface with respect to the ambient temperature $T_{\infty}$ in case (ii), its excess temperature at leading edge in case (i) and at the distance $x=r_{0}$ in case (iii), respectively. In case (i) the exponent $a$ represents the logarithmic increment of the surface temperature with respect to $T_{0}$, i.e.,

$$
a=\ln \left[\frac{T_{w}\left(r_{0}\right)-T_{\infty}}{T_{0}}\right] .
$$

It is worth mentioning here that the mixed convection counterpart of the present problem for clear fluids with the stream velocity and surface temperature distributions $\left\{U(x), T_{w}(x)\right\}$ corresponding to our case (iii) has comprehensively been investigated by Mahmood and Merkin (1988).

\section{Derivation of the Exactly Solvable Cases}

The axial velocity component $u=U(x)$ being known, the continuity equation (1) can be integrated once with respect to $r$ and thus, along with the impermeability condition $v=0$ on $r=r_{0}$, it yields for the radial velocity component the expression 


$$
v=-\frac{r^{2}-r_{0}^{2}}{2 r} \frac{\mathrm{d} U}{\mathrm{~d} x}
$$

This equation shows that in case (i) the radial velocity component is zero, i.e., we are faced there with a "parallel flow". In the other two cases, $v$ is non-vanishing (except on the cylinder surface $r=r_{0}$ ).

With the velocity components $(u, v)$ given by Eqs. (2) and (9), the pressure field can also be obtained by a further integration of the Darcy equations $u=-(K / \mu)(\partial p / \partial x)$, $v=-(K / \mu)(\partial p / \partial r)$ yielding the result

$$
p=p_{0}-\frac{\mu}{K} \int_{0}^{x} U \mathrm{~d} x+\frac{\mu}{2 K}\left[\frac{r^{2}-r_{0}^{2}}{2}-r_{0}^{2} \ln \left(\frac{r}{r_{0}}\right)\right] .
$$

Here $p_{0}$ is the pressure at $(x, r)=\left(0, r_{0}\right)$.

Bearing in mind the thermal boundary conditions, we are looking for the temperature solution in the form

$$
T=T_{\infty}+\left[T_{w}(x)-T_{\infty}\right] \theta(\eta)
$$

where

$$
\eta=\frac{\sqrt{P e}}{2 r_{0}^{2}}\left(r^{2}-r_{0}^{2}\right)
$$

is a new independent variable, and

$$
P e=\frac{U_{0} r_{0}}{\alpha_{m}}
$$

is the Péclet number. As a consequence of Eqs. (11) and (12), the thermal boundary conditions (4) become

$$
\theta(0)=1, \quad \theta(\infty)=0
$$

We mention that the dependence of the new variable $\eta$ on the radial coordinate in the quadratic form $\left(r^{2}-r_{0}^{2}\right)$ is suggested by the work of Minkowycz and Cheng (1976) in which it has been shown that the free convection problem on a vertical cylinder admits an exact similarity reduction when (and only when) the surface temperature distribution is a linear function of the axial coordinate [as in our case (iii)]. In this case, the pertinent similarity variable depends on $\left(r^{2}-r_{0}^{2}\right)$ only.

Now, applying the transformations $(11,12)$ and bearing in mind the expressions $(2)$ and (9) of the velocity components $u$ and $v$, the temperature equation (3) goes over in

$$
\left(1+\frac{2 \eta}{\sqrt{P e}}\right) \frac{\mathrm{d}^{2} \theta}{\mathrm{d} \eta^{2}}+\left(\frac{2}{\sqrt{P e}}+\frac{\mathrm{d} U}{\mathrm{~d} x} \frac{r_{0}}{U_{0}} \eta\right) \frac{\mathrm{d} \theta}{\mathrm{d} \eta}-\frac{U}{T_{w}-T_{\infty}} \frac{\mathrm{d}\left(T_{w}-T_{\infty}\right)}{\mathrm{d} x} \frac{r_{0}}{U_{0}} \theta=0 .
$$

A simple inspection of this equation (which in general is still a partial differential equation involving the variables $\eta$ and $x$ ) shows that there exist exactly four cases of the distributions $\left\{U(x), T_{w}(x)\right\}$ for which Eq. (15) can be reduced to an ordinary differential equation involving only the transformed radial variable $\eta$. Three of them are precisely our cases (i)(iii) specified by Eqs. (5)-(7), while the fourth one corresponds to the uniform velocity and 
uniform surface temperature distributions $\left\{U(x), T_{w}(x)\right\}=\left\{U_{0}, T_{\infty}+T_{0}\right\}$. In the latter case, Eq. (15) reduces to

$$
\frac{\mathrm{d}}{\mathrm{d} \eta}\left[\left(1+\frac{2 \eta}{\sqrt{P e}}\right) \frac{\mathrm{d} \theta}{\mathrm{d} \eta}\right]=0 .
$$

However, the general solution

$$
\theta=C_{1}+C_{2} \ln \left(1+\frac{2 \eta}{\sqrt{P e}}\right)
$$

of this equation cannot satisfy both the boundary conditions (14). Obviously, this result does not mean that for the uniform distributions $\left\{U(x), T_{w}(x)\right\}=\left\{U_{0}, T_{\infty}+T_{0}\right\}$, i.e., for an isothermal cylinder in a uniform stream, no solution exists. It only means that in this case no solution of the separable form $(11,12)$ exists. This conclusion is in full agreement with the forced convection study of Vasantha et al. (1987) who showed that in the uniform case $\left\{U(x), T_{w}(x)\right\}=\left\{U_{0}, T_{\infty}+T_{0}\right\}$ the temperature field has to be described by a non-separable function $T=T_{\infty}+\left(T_{w}-T_{\infty}\right) \theta(x, r)$.

It is easy to see that in our cases (i)-(iii), Eq. (15) reduces to the following ordinary differential equations

$$
\begin{gathered}
\text { Case (i): } \quad\left(1+\frac{2 \eta}{\sqrt{P e}}\right) \frac{\mathrm{d}^{2} \theta}{\mathrm{d} \eta^{2}}+\frac{2}{\sqrt{P e}} \frac{\mathrm{d} \theta}{\mathrm{d} \eta}-a \theta=0 \\
\text { Case (ii): }\left(1+\frac{2 \eta}{\sqrt{P e}}\right) \frac{\mathrm{d}^{2} \theta}{\mathrm{d} \eta^{2}}+\left(\frac{2}{\sqrt{P e}}+\eta\right) \frac{\mathrm{d} \theta}{\mathrm{d} \eta}=0 \\
\text { Case (iii): } \quad\left(1+\frac{2 \eta}{\sqrt{P e}}\right) \frac{\mathrm{d}^{2} \theta}{\mathrm{d} \eta^{2}}+\left(\frac{2}{\sqrt{P e}}+\eta\right) \frac{\mathrm{d} \theta}{\mathrm{d} \eta}-\theta=0 .
\end{gathered}
$$

In all these cases, the boundary conditions are given by the same Eq. (14).

\section{The Exact Solutions}

In this section, the solutions of the boundary value problems $(18,14),(19,14)$ and $(20,14)$ will be given in an exact analytical form.

\section{Case (i)}

With the aid of the variable transformation

$$
\xi=\sqrt{a P e\left(1+\frac{2 \eta}{\sqrt{P e}}\right)}
$$

the governing equation (18) reduces to the equation of the modified Bessel functions $I_{\nu}(\xi)$ and $K_{v}(\xi)$ for $v=0$ (see Eq. 9.6.1 of Abramowitz and Stegun 1972). Thus the solution of the problem $(18,14)$, is obtained as

$$
\theta=\frac{K_{0}\left(\sqrt{a P e\left(1+\frac{2 \eta}{\sqrt{P e}}\right)}\right)}{K_{0}(\sqrt{a P e})}=\frac{K_{0}\left(\sqrt{a P e} \frac{r}{r_{0}}\right)}{K_{0}(\sqrt{a P e})} .
$$


The corresponding surface temperature gradient in the radial direction is given by

$$
\left.\frac{\mathrm{d} \theta}{\mathrm{d} r}\right|_{r=r_{0}}=-\frac{\sqrt{a P e}}{r_{0}} \frac{K_{1}(\sqrt{a P e})}{K_{0}(\sqrt{a P e})}
$$

It is worth emphasizing here that (for a fixed $r_{0}$ ) the solution (22) depends only on the product $a P e$ of the temperature exponent $a$ and the Péclet number. Thus, the temperature profiles (22) as functions of $r / r_{0}$ will collapse down in a single curve for all values of $a$ and $P e$ for which their product takes the same value.

\section{Case (ii)}

The governing equation (19) can be integrated twice, and thus the exact solution of the problem $(19,14)$ can be given in terms of the incomplete Gamma function $\Gamma(a, x)$ (see Eq. 6.5.3 of Abramowitz and Stegun 1972) in the form

$$
\theta=\frac{\Gamma\left(\frac{P e}{4}, \frac{P e}{4}+\frac{\sqrt{P e}}{2} \eta\right)}{\Gamma\left(\frac{P e}{4}, \frac{P e}{4}\right)}=\frac{\Gamma\left(\frac{P e}{4}, \frac{P e}{4} \frac{r^{2}}{r_{0}^{2}}\right)}{\Gamma\left(\frac{P e}{4}, \frac{P e}{4}\right)} .
$$

The corresponding surface temperature gradient in the radial direction is given by

$$
\left.\frac{\mathrm{d} \theta}{\mathrm{d} r}\right|_{r=r_{0}}=-\frac{2 \mathrm{e}^{\frac{P e}{4}}}{r_{0} \Gamma\left(\frac{P e}{4}, \frac{P e}{4}\right)}\left(\frac{P e}{4}\right)^{\frac{P e}{4}} .
$$

\section{Case (iii)}

In order to solve the boundary value problem $(20,14)$, it is convenient to change from $\eta$ to a further independent variable $z$ defined as

$$
z=-\frac{P e}{4}-\frac{\sqrt{P e}}{2} \eta
$$

By doing so, the governing equation (20) reduces to the differential equation of Kummer's confluent hypergeometric functions $M(a, b, z)$ and $U(a, b, z)$ (see Eq. 13.1.1 of Abramowitz and Stegun (1972)) with the parameters $a$ and $b$ given by

$$
a=-1, \quad b=1-\frac{P e}{4} .
$$

Accordingly, the general solution of Eq. (20) can be written as the linear combination

$$
\theta=C_{1} \mathrm{e}^{z} M(b-a, b,-z)+C_{2} \mathrm{e}^{z} U(b-a, b,-z) .
$$

However, the first term of Eq. (28) would violate the asymptotic condition $\theta(\eta) \rightarrow 0$ as $\eta \rightarrow$ $\infty$ which thus requires $C_{1}=0$. Therefore the temperature solution which satisfies the boundary conditions (14) reads

$$
\theta=\frac{U\left(2-\frac{P e}{4}, 1-\frac{P e}{4}, \frac{P e}{4}+\frac{\sqrt{P e}}{2} \eta\right)}{U\left(2-\frac{P e}{4}, 1-\frac{P e}{4}, \frac{P e}{4}\right)} \mathrm{e}^{-\frac{\sqrt{P e}}{2} \eta} .
$$


In terms of the dimensionless radial variable $r / r_{0}$, this solution has the explicit form

$$
\theta=\frac{U\left(2-\frac{P e}{4}, 1-\frac{P e}{4}, \frac{P e}{4} \frac{r^{2}}{r_{0}^{2}}\right)}{U\left(2-\frac{P e}{4}, 1-\frac{P e}{4}, \frac{P e}{4}\right)} \exp \left[-\frac{P e}{4}\left(\frac{r^{2}}{r_{0}^{2}}-1\right)\right] .
$$

Using the property $U^{\prime}(a, b, x)=U(a, b, x)-U(a, b+1, x)$ of the $x$-derivative of $U$ (see Eq. 13.4.25 of Abramowitz and Stegun 1972), we obtain for the wall- temperature gradient in the radial direction the expression

$$
\left.\frac{\mathrm{d} \theta}{\mathrm{d} r}\right|_{r=r_{0}}=-\frac{P e}{2 r_{0}} \frac{U\left(2-\frac{P e}{4}, 2-\frac{P e}{4}, \frac{P e}{4}\right)}{U\left(2-\frac{P e}{4}, 1-\frac{P e}{4}, \frac{P e}{4}\right)} .
$$

For the special values $P e=8 n, n=1,2,3, \ldots$ of the Péclet number, the solution (29) can be expressed in term of the generalized Laguerre polynomials $L_{N}^{(\alpha)}(x)$ (see Chapt. 22 of Abramowitz and Stegun 1972) as

$$
\theta=\frac{L_{2 n-2}^{(-2 n)}(2 n+\sqrt{2 n} \eta)}{L_{2 n-2}^{(-2 n)}(2 n)} \mathrm{e}^{-\sqrt{2 n} \eta}
$$

The generalized Laguerre polynomials $L_{N}^{(\alpha)}(x)$ can easily be calculated with the aid of the Rodriguez formula

$$
L_{N}^{(\alpha)}(x)=\frac{x^{-\alpha} \mathrm{e}^{x}}{N !} \frac{\mathrm{d}^{N}}{\mathrm{~d} x^{N}}\left(x^{\alpha+N} \mathrm{e}^{-x}\right) .
$$

For example, for $n=1$ and $n=2$ (i.e., for $P e=8$ and $P e=16$ ) one obtains

$$
\theta=\mathrm{e}^{-\sqrt{2} \eta} \text { and } \theta=\frac{1}{19}\left(19+12 \eta+2 \eta^{2}\right) \mathrm{e}^{-2 \eta},
$$

respectively. We mention that the solution $\theta=\mathrm{e}^{-\sqrt{2} \eta}$ corresponding to $P e=8$ coincides with the forced convection case of the special mixed convection solution reported by Magyari et al. (2005).

\section{Curvature Effects}

The quantity

$$
c \equiv \frac{2}{\sqrt{P e}}=\sqrt{\frac{4 \alpha_{m}}{r_{0} U_{0}}}
$$

occurring in the governing equations (18)-(20) approaches zero when the cylinder radius $r_{0}$ goes to infinity. Thus from geometrical point of view, $c$ can be interpreted as a curvature parameter of the cylinder. In this limiting case, Eqs. (18)-(20) reduce to

$$
\begin{gathered}
\text { Case (i): } \frac{\mathrm{d}^{2} \theta}{\mathrm{d} \eta^{2}}-a \theta=0\left(r_{0} \rightarrow \infty\right) \\
\text { Case (ii): } \frac{\mathrm{d}^{2} \theta}{\mathrm{d} \eta^{2}}+\eta \frac{\mathrm{d} \theta}{\mathrm{d} \eta}=0\left(r_{0} \rightarrow \infty\right)
\end{gathered}
$$




$$
\text { Case (iii): } \frac{\mathrm{d}^{2} \theta}{\mathrm{d} \eta^{2}}+\eta \frac{\mathrm{d} \theta}{\mathrm{d} \eta}-\theta=0\left(r_{0} \rightarrow \infty\right) .
$$

Therefore, Eqs. (36)-(37) describe forced convection thermal boundary layers on plane surfaces corresponding to the velocity and surface temperature and distributions $\left\{U(x), T_{w}(x)\right\}$ specified by Eqs. (5)-(7). This is the reason why, e.g., the above Eq. (38) coincides with Cheng's equation (29) (Cheng 1977), which describes the forced convection temperature field on a plane surface with linear temperature distribution $T_{w}-T_{\infty}=A x$ in the background flow $(u, v)=(B x,-B y)$.

The solutions of Eqs. (36)-(38) are

$$
\begin{gathered}
\text { Case (i): } \theta=\mathrm{e}^{-\sqrt{a} \eta}\left(r_{0} \rightarrow \infty\right) \\
\text { Case (ii): } \theta=\operatorname{erfc}\left(\frac{\eta}{\sqrt{2}}\right)\left(r_{0} \rightarrow \infty\right) \\
\text { Case (iii): } \theta=\mathrm{e}^{-\frac{\eta^{2}}{2}}-\sqrt{\frac{\pi}{2}} \eta \operatorname{erfc}\left(\frac{\eta}{\sqrt{2}}\right)\left(r_{0} \rightarrow \infty\right) .
\end{gathered}
$$

\section{Heat Transfer Characteristics}

The dimensionless heat transfer characteristic of the flow is the Nusselt number

$$
N u=\frac{q_{w} r_{0}}{k\left(T_{w}-T_{\infty}\right)}, \quad q_{w}=-\left.k \frac{\partial T}{\partial r}\right|_{r=r_{0}},
$$

where $q_{w}$ is the radial heat flux across the cylinder surface. Thus with the aid of Eq. (11), we obtain

$$
N u=-\left.r_{0} \frac{\partial \theta}{\partial r}\right|_{r=r_{0}} .
$$

Now bearing in mind Eqs. (23), (25), and (31), we obtain for the Nusselt numbers of the three exactly solvable cases

$$
\begin{aligned}
N u_{(i)} & =\sqrt{a P e} \frac{K_{1}(\sqrt{a P e})}{K_{0}(\sqrt{a P e})} \\
N u_{(i i)} & =\frac{2 \mathrm{e}^{-\frac{P e}{4}}}{\Gamma\left(\frac{P e}{4}, \frac{P e}{4}\right)}\left(\frac{P e}{4}\right)^{\frac{P e}{4}} \\
N u_{(i i i)} & =\frac{P e}{2} \frac{U\left(2-\frac{P e}{4}, 2-\frac{P e}{4}, \frac{P e}{4}\right)}{U\left(2-\frac{P e}{4}, 1-\frac{P e}{4}, \frac{P e}{4}\right)} .
\end{aligned}
$$

For the special values $P e=8 n, n=1,2,3, \ldots$, the expression (46) of the Nusselt number reduces to

$$
N u_{(i i i)}=4 n \frac{L_{2 n-2}^{(1-2 n)}(2 n)}{L_{2 n-2}^{(-2 n)}(2 n)}
$$

For example, Eq. (47) yields $N u_{(i i i)}=4$ for $n=1$ and $N u_{(i i i)}=104 / 19$ for $n=2$. 
Table 1 Behavior of the Nusselt number at small and large values of the Péclet number in the exactly solvable cases

\begin{tabular}{lc}
\hline$P e \rightarrow 0$ & $P e \rightarrow \infty$ \\
\hline$N u_{(i)}=\frac{2}{\left|2 \gamma+\ln \left(\frac{a P e}{4}\right)\right|}$ & $N u_{(i)}=\sqrt{a P e}$ \\
$N u_{(i i)}=\frac{2}{\left|\gamma+\ln \left(\frac{P e}{4}\right)\right|}$ & $N u_{(i i)}=\sqrt{\frac{2 P e}{\pi}}$ \\
$N u_{(i i i)}=\frac{2}{\left|1+\gamma+\ln \left(\frac{P e}{4}\right)\right|}$ & $N u_{(i i i)}=\sqrt{\frac{\pi P e}{2}}$ \\
\hline
\end{tabular}

Here $\gamma$ denotes the Euler constant, $\gamma=0.577216$. In case (i), the limiting behaviors for $a P e \rightarrow 0$ and $a P e \rightarrow \infty$ have been considered

In the limiting case of the plane surfaces $\left(r_{0} \rightarrow \infty\right)$, the Nusselt numbers associated with the respective temperature fields (39)-(41) are

$$
N u_{(i)}=\sqrt{a P e}, \quad N u_{(i i)}=\sqrt{\frac{2 P e}{\pi}}, \quad N u_{(i i i)}=\sqrt{\frac{\pi P e}{2}}\left(r_{0} \rightarrow \infty\right) .
$$

\section{Discussion}

We first notice that according to the definition (13) of the Péclet number, $r_{0} \rightarrow \infty$ implies also $P e \rightarrow \infty$. We thus arrive to the conclusion that Eqs. (48) describe at the same time the scaling behavior of the Nusselt number for large values of the Péclet number. It is also worth emphasizing that in all three cases, the Nusselt number scales with $(P e)^{1 / 2}$ as $P e \rightarrow \infty$. A simple dependence of the Nusselt number on the Péclet number can also be found for $\mathrm{Pe} \rightarrow 0$ by using the properties of the transcendental functions involved in the respective expressions (44)-(46). The results of these estimations are included, in addition to the large$P e$ formulas (48), in Table 1. The truth of all the relationships collected in Table 1 can also be "proved" numerically, comparing them to the respective exact expressions (44)-(46).

It is interesting to notice, that in contrast to the scaling behavior of the Nusselt number for $P e \rightarrow \infty$, in the limiting range $P e \rightarrow 0$ no power-law dependence on $P e$ exists. In all three cases, the Nusselt number approaches zero as $P e \rightarrow 0$ according to the reciprocal logarithmic law $N u \sim|\ln (P e)|^{-1}$. In the intermediary range of $P e$, the functional dependence $N u=N u(P e)$ is much more complicated and is described by the exact results (44)-(46). As an illustration, in Fig. 2, the Nusselt numbers (i)-(iii) given by Eqs. (44)-(46) have been plotted as functions of the Péclet number in the range $0 \leq P e \leq 100$. To be specific, in case (i) for the logarithmic increment of the exponential surface, temperature distribution the value $a=3$ has been chosen.

The circumstance that in case (i) the Nusselt number (44) depends only on the product $a P e$, makes the comparison of $N u_{(i)}$ to the other two curves of Fig. 2 somewhat arbitrary. However, it is just this mathematical degeneracy of $N u_{(i)}$ with respect to $a$ and $P e$ which makes the issue physically interesting. Indeed, a simple inspection of the second column of Table 1 shows that for large values of the Péclet number, the curve $N u_{(i)}$ of Fig. 2 corresponding to the exponential surface temperature distribution (5) can be superimposed on the curve of $N u_{(i i)}$ corresponding to the constant surface temperature (6) for $a=2 / \pi=0.6366$ and on the curve of $N u_{(i i i)}$ corresponding to the linear surface temperature distribution (7) for $a=\pi / 2=1.5708$. Similar knowledge can be gained from the first column of 


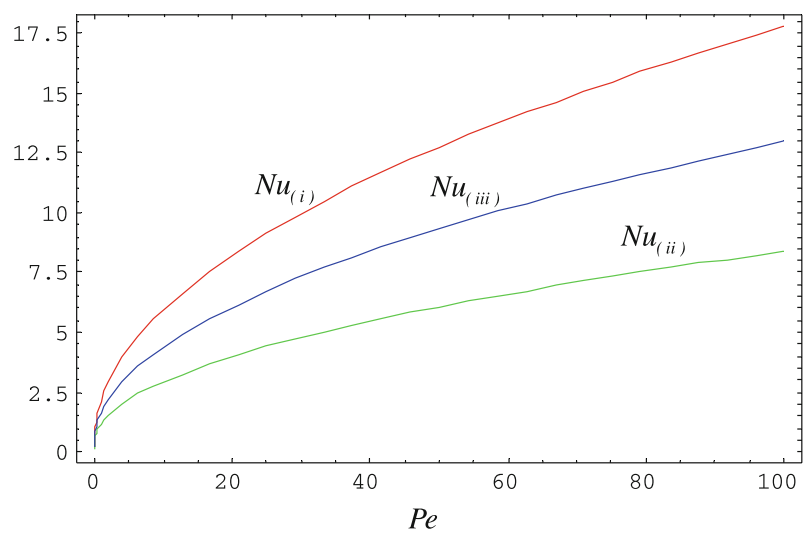

Fig. 2 Plot of the Nusselt numbers as a function of the Péclet number for the exactly solvable cases (i)-(iii). In case (i), $a=3$ has been chosen

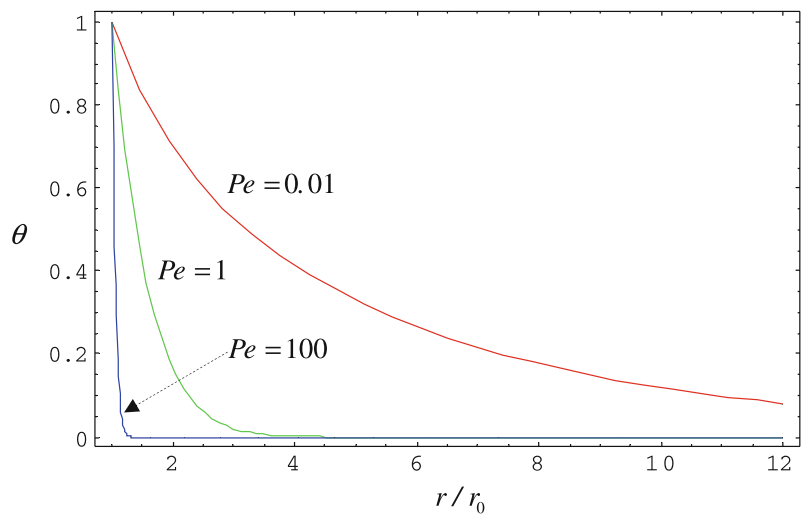

Fig. 3 Temperature profiles (30) corresponding to the linear surface temperature distribution (7) plotted as functions of $r / r_{0}$ for the three indicated values of the Péclet number

Table 1. Namely, in the range $P e \rightarrow 0, N u_{(i)}$ can be superimposed on the curve of $N u_{(i i)}$ for $a=\mathrm{e}^{-\gamma}=0.5614$ and on the curve of $N u_{(i i i)}$ for $a=\mathrm{e}^{1-\gamma}=1.5262$.

The temperature profiles (22), (24), and (30) corresponding to the three exactly solvable cases are all monotonically decreasing functions of the dimensionless radial coordinate $r / r_{0}$. This result is physically expected, since the wall temperature is either constant [case (ii)] or a monotonically increasing function of the axial distance $x$ [cases (i) and (iii)], so that for a temperature overshoot in the fluid no reason exists. It also turns out that the larger $P e$, the steeper the temperature profiles (22), (24), and (30) which is in a full agreement with Fig. 2. These features are illustrated in Fig. 3, where the temperature profiles (3) corresponding to the case (iii) have been plotted for three selected values of the Péclet number $r / r_{0}$.

\section{Summary and Conclusions}

Three exactly solvable cases of the porous medium forced convection heat transfer from a heated cylinder in a longitudinal background flow have been described in this paper. 
It has been shown that the velocity distribution $U=U(x)$ of the axial driving stream and the surface temperature distribution $T_{w}=T_{w}(x)$ associated with these cases are (i) $\left\{U(x), T_{w}(x)\right\}=\{$ uniform, exponential $\}$, (ii) $\left\{U(x), T_{w}(x)\right\}=\{$ linear, uniform $\}$, and (iii) $\left\{U(x), T_{w}(x)\right\}=\{$ linear, linear $\}$, respectively. The exact solutions are always of the separable form $T(x, r)-T_{\infty}=\left(T_{w}(x)-T_{\infty}\right) \theta(\eta)$, where $\eta$ depends only on the radial coordinate $r$, and have been given in the paper in terms of the modified Bessel function, the incomplete Gamma function and the confluent hypergeometric functions, respectively. Thus the dependence of the Nusselt number on the Péclet number as well as the curvature effects could be described exactly. It is worth emphasizing again that:

- In the limiting range of small Péclet numbers, in all three cases, a reciprocal logarithmic behavior of the Nusselt number has been found, $N u \sim|\ln (P e)|^{-1}$, while in the range of large $P e$ 's, a square root behavior $N u \sim(P e)^{1 / 2}$ occurs.

- The effect of a strongly curved surface $\left(r_{0} \rightarrow 0\right)$ on the Nusselt number occurs there as a "small $P e$-effect", while a small curvature $\left(r_{0} \rightarrow \infty\right)$ of the cylinder surface as a "large $P e$-effect" is perceived.

- Compared to the other two surface temperature distributions, the exponential distribution of case (i). $T_{w}(x)-T_{\infty}=T_{0} \exp \left(a x / r_{0}\right)$, possesses some "universal" features. Namely, for certain values of the logarithmic increment $a$, the curve $N u(P e)$ of the corresponding Nusselt number $N u_{(i)}$ can be superimposed on the curves of $N u_{(i i)}$ and $N u_{(i i i)}$, of the other two cases, respectively, both for $P e \rightarrow 0$ and $P e \rightarrow \infty$, respectively.

\section{References}

Abramowitz, M., Stegun, I. S.: Handbook of Mathematical Functions. Dover, New York (1972)

Bassom, A. P., Rees, D. A. S.: Free convection from a heated vertical cylinder embedded in a fluid-saturated porous medium. Acta Mech. 116, 139-151 (1996)

Cheng, P.: Combined free and forced convection flow about inclined surfaces in porous media. Int. J. Heat Mass Transf. 20, 807-814 (1977)

Hooper, W. B., Chen, T. S., Armaly, B. F.: Mixed convection along an isothermal vertical cylinder in porous media. AIAA J. Thermophys. Heat Transf. 8, 92-99 (1994)

Magyari, E., Pop, I., Keller, B.: Exact solutions for a longitudinal steady mixed convection flow over a permeable vertical cylinder in a porous medium. Int. J. Heat Mass Transf. 48, 3435-3442 (2005)

Mahmood, T., Merkin, J. H.: Similarity solutions in axisymmetric mixed-convection boundary-layer flow. J. Eng. Math. 22, 73-92 (1988)

Merkin, J. H., Pop, I.: Mixed convection boundary-layer on a vertical cylinder embedded in a saturated porous medium. Acta Mech. 66, 251-262 (1987)

Minkowycz, W. J., Cheng, P.: Free convection about a vertical cylinder embedded in a porous medium. Int. J. Heat Mass Transf. 19, 805-813 (1976)

Nield, D. A., Bejan, A.: Convection in Porous Media. 3rd edn. Springer, New York (2006)

Pop, I., Na, T.-J.: Darcian mixed convection along slender vertical cylinders with variable surface heat flux embedded in a porous medium. Int. Commun. Heat Mass Transf. 25, 251-260 (1998)

Vasantha, R., Nath, G., Pop, I.: Forced convection along a longitudinal cylinder embedded in a saturated porous medium. Int. Commun. Heat Mass Transf. 14, 639-646 (1987) 